Technologiekonkurrenz, des sich ausweitenden Welthandels, der fortschreitenden militärtechnologischen Revolution, ungelöster regionaler Konflikte und fehlender Konfliktlösungsmechanismen vor völlig neuen Herausforderungen steht. Dabei müssen sowohl die Erfolge der bisherigen Rüstungskontrollbemühungen gegen machtpolitische Alleingänge der USA gesichert werden, als auch die neuen Herausforderungen durch die zunehmende Kommerzialisierung und Privatisierung von Rüstung und Sicherheit bewältigt werden.

Internationale und regionale Abkommen zur Kontrolle und Abrüstung der vorhandenen Waffenarsenale verbunden mit der Einhaltung und gegebenenfalls Verschärfung von Rüstungsexportrichtlinien bleiben unverzichtbare Instrumente, um der Verbreitung von Massenvernichtungswaffen und konventionellen Waffen aller Art Einhalt zu gebieten. Die Behauptung, die klassischen Instrumente der Rüstungskontrolle funktionierten nicht mehr, ist ein wohlfeiles Argument, das in Washington bereits Anfang der neunziger Jahre formuliert wurde: Rüstungskontrolle und Verifikation wird es niemals flächendeckend und allumfassend geben. Auch künftig wird es Möglichkeiten geben, Rüstungskontrollverträge und die darin enthaltenen Kontroll- und Überprüfungsmechanismen zu umgehen bzw. zu unterlaufen. Gleichwohl gibt es zur vertragsbasierten und verifizierbaren Rüstungskontrolle nur eine Alternative: ein weltweites nukleares, chemisches und biologisches Wettrüsten. Ein solches kann auch nicht im Interesse der USA liegen. Die Risiken, die aus der Trias der Bedrohung durch transnationalen Terrorismus, Massenvernichtungswaffen und zerfallende Staaten resultieren, sind jedenfalls mit nachrichtendienstlichen, rüstungskontrollpolitischen und polizeilichen Instrumentarien wirksamer $\mathrm{zu}$ bekämpfen als durch militärische Interventionen.

\title{
Rüstungskontrolle in Asien: Regionale Konzepte und ihre Erfolgsbedingungen
}

\begin{abstract}
Dr. Christopher Daase, Lecturer in International Relations, University of Kent at Canterbury, Brussels School of International Studies, Brüssel/ Prof. Dr. Susanne Feske, Institut für Politikwissenschaft, Westfälische Wilhelms-Universität Münster.
\end{abstract}

terogenität Asiens, die das Problem der Rüstungsdynamik ungleich komplexer macht als dies in Europa oder generell in der nördlichen Hemisphäre der Fall ist. Genauso wichtig ist allerdings die Abneigung gegen Konzepte, die als vom Westen den asiatischen Staaten aufgezwungen empfunden werden: Was für Europa richtig war oder ist, muss für Asien nicht auch richtig sein. ${ }^{1}$ Diese Empfindlichkeit ist Ausdruck historisch begründeter Erfahrungen während der Kolonialzeit; aber auch in der Gegenwart wird von asiatischer Seite immer wieder argumentiert, dass der sich als überlegen fühlende Westen weiterhin versucht, die südliche Hemisphäre zu dominieren und ihr das eigene Werte- und Normensystem und letztlich auch die eigenen Problemlösungsstrategien überzustülpen. Drittens werden auch kulturelle Argumente ins Feld geführt, die erklären sollen, warum westliche Rüstungskontrollkonzepte für Asien ungeeignet sind. Die Verhandlungs- und Kooperationskultur in Asien weist nämlich Besonderheiten auf, die in westlichen Konzepten keine Rolle spielen. Ein zirkuläres Zeitverständnis in Asien führt beispielsweise dazu,

1 Vgl. Gill Bates/J.M. Mak, Introduction, in: Dies., (Hrsg.), Arms, Transparency and Security in Southeast Asia, Oxford 1997, 1-9, 2. dass Tagesordnungen oder Verhandlungsabläufe anders organisiert werden als in westlichen Modellen, denen ein lineares Zeitverständnis zugrundeliegt. Außerdem spielt in Asien der Konsensgedanke eine wichtige Rolle. Es geht deshalb nicht in erster Linie darum, die eigene bargaining power zu erhöhen und Maximalforderungen durchzusetzen. Ein gutes Ergebnis aus asiatischer Sicht berücksichtigt vielmehr die legitimen Interessen aller Beteiligten und sollte diese miteinander in Einklang bringen. In der Praxis bedeutet dies, dass Verhandlungen weniger ergebnisorientiert geführt und Erfolge nicht unbedingt Schritt für Schritt als chronologischer Fortschritt erzielt werden. Das Ziel von Verhandlungen ist nicht notwendigerweise eine schriftliche Vereinbarung, die unterschrieben und ratifiziert wird. Verhandlungsprozesse haben in Asien durchaus einen Selbstzweck, insofern sie die Kommunikation zwischen Antagonisten ermöglichen und das harmonische Aushandeln und Abgleichen von Postitionen gestatten. Auch hierin unterscheidet sich die westliche Vertragskultur von der asiatischen Verhandlungskultur.

Angesichts dieses Widerwillens, auf Theorien und Konzepte der Rüstungskontrolle zurückzugreifen, die während des Kalten Krieges in Europa und den Vereinigten Staaten entwickelt worden sind, ${ }^{2}$ ist es notwendig, nach indigenen Ansätzen und Ideen kooperativer Rüstungssteuerung in Asien zu suchen

2 Vgl. dazu insbesondere Jozef Goldblat, Arms Control: A Guide to Negotiations and Agreements, London 1994; Stuart Croft, Strategies of Arms Control: A History and Typology, Manchester 1996; Michael O. Wheeler, A History of Arms Control, in: Jeffrey A. Larsen, (Hrsg.), Arms Control. Cooperative Security in a Changing Environment, Boulder 2002, 19-39. 
und sie auf ihre Stärken und Schwächen hin zu prüfen. Was für genuin asiatische Ansätze der Rüstungskontrolle gibt es? Welche Resultate haben sie bislang erbracht? Und wo lassen sich Defizite erkennen? Bei der Beurteilung ist es allerdings wichtig, Effizienz und Effektivität nicht nur am erzielten Output zu bemessen, sondern den Prozess der Verhandlungen einzuschätzen. Welche Akteure und Konfliktparteien waren daran beteiligt und welche Probleme wurden thematisiert? Welche Entwicklungsmöglichkeiten haben bisherige Verhandlungen eröffnet?

Dabei müssen drei Sub-Regionen innerhalb Asiens unterschieden werden, die völlig unterschiedliche Rüstungsdynamiken und politische Rahmenbedingungen aufweisen:

- Südasien: Indien und Pakistan haben 1998 Nuklearwaffen getestet. Beide Staaten sind in Territorialkonflikte miteinander verwickelt, die bis in die jüngste Vergangenheit militärisch ausgetragen wurden.

- Südostasien: Nach dem Ende des Kalten Krieges ist zwar die Blockkonfrontation zwischen den Indochina-Staaten und den übrigen sechs südostasiatischen Staaten aufgehoben, dennoch setzt sich der Rüstungswettlauf im konventionellen Bereich weiter fort. Zudem liegt Südostasien im Schnittpunkt von drei Nuklearmächten (China, Russland und den USA).

- Nordostasien: Während der Nuklearkonflikt um Nordkorea die Schlagzeilen bestimmt, bestehen die zahlreichen Territorialkonflikte in der Region fort. Rüstungskooperation zwischen den USA, Taiwan, Südkorea und Japan im Bereich der Raketenabwehr sowie Rüstungsexporte von China und Nordkorea haben zu politischen Spannungen geführt.

Der Bedarf an Rüstungskontrolle in Asien ist also groß. Alte Konflikte müssen entschärft und neue, sich abzeichnende Krisen kooperativ beweltigt werden. ${ }^{3}$ Doch die Voraussetzungen dafür sind in den drei Regionen sehr unterschiedlich. Im folgenden werden die subregionalen Konfliktkonstellationen skizziert und die indigenen Ansätze zur Rüstungskontrolle insbesondere hinsichtlich ihrer institutionellen Grundlagen analysiert. Denn unabhängig davon, ob man »Institutionalisierung « für ein westliches Konzept hält oder nicht: Die Entwicklung tragfähiger Kooperationstrukturen wird auch in Zukunft der Schlüssel für den Erfolg asiatischer Rüstungskontrolle sein.

\section{Südasien: Eingebettete Bipolaritiät}

Im Mai 1998 testete zunächst Indien, kurze Zeit später auch Pakistan, Nuklearwaffen. Diese Tests riefen weltweit Empörung hervor, auch wenn keiner der beiden Staaten geltendes internationales Recht gebrochen hatte: Beide sind weder Mitglieder des Atomwaffensperrvertrages (Nonproliferation Treaty $=$ NPT) noch haben sie den Umfassenden Test-Stopp-Vertrag (Comprehensive Test Ban Treaty = CTBT) unterzeichnet. Die Kritiker der Nukleartests sahen trotzdem das globale Nichtverbreitungsregime beschädigt, das nur den fünf ständigen Mitgliedern des Weltsicherheitsrates den Besitz

3 Nicholas Berry, Fertile Ground for Arms Control in Asia, in: CDI Asia Forum, 8 December 2000 (http://www.cdi.org/asia/fa12800.html). von Nuklearwaffen gestattet. Darüber hinaus sahen sie die regionale Sicherheitslage in Südasien dramatisch destabilisiert. Befürworter der Tests argumentierten demgegenüber, dass der offene Nuklearwaffenbesitz einer »opaken Proliferation « vorzuziehen sei, weil er die Gefahr von Fehleinschätzung und unautorisiertem Einsatz minimiere. ${ }^{4}$ Ferner sei nur auf der Grundlage transparenter nuklearer Fähigkeiten der Aufbau eines stabilen regionalen Abschreckungssystems möglich; und erst auf der Grundlage stabiler Abschreckung könnten weitergehende Rüstungskontroll- und Abrüstungsschritte eingeleitet werden.

Die Ereignisse seit 1998 geben beiden Positionen Recht. Einerseits verschärfte sich der Territorialkonflikt über Kaschmir, als 1999, nur wenige Wochen nach dem historischen Treffen zwischen dem indischen Premierminister Vajpayee und dem pakistanischen Premierminister Sharif in Lahore, pakistanische Soldaten indisches Territorium in Kagil besetzten. Die anschließenden Kämpfe des vierten indisch-pakistanischen Krieges kosteten auf beiden Seiten etwa je 1.000 Soldaten das Leben. ${ }^{5}$ Andererseits wurden weder in Kigali noch während der Krise im Sommer 2002, als Indien Pakistan vorwarf, es würde Taliban- und El-Kaida-Kämpfer für die »Befreiung « Kaschmirs rekrutieren, Nuklearwaffen eingesetzt: Eine unkontrollierte Eskalation konnte vermieden werden. Damit scheint sich zu bestätigen, was Glenn Snyder schon 1961 das »Stabilitäts-Instabilitäts-Paradox « genannt hatte, nämlich die Tatsache, dass durch glaubhafte nukleare Drohung ein Anreiz zu nicht-nuklearem Konfliktaustrag entsteht, weil beide Akteure annehmen, der Gegner werde die Kampfhandlungen nicht zum äußersten eskalieren lassen. ${ }^{6}$

Während Pessimisten argumentieren können, dass die Krise 2002 leicht zu einem nuklearen Schlagabtausch hätte führen können, wenn nicht die internationale Gemeinschaft eingeschritten wäre und die Kontrahenten zur Vernunft ermahnt hätte, können Optimisten darauf verweisen, dass eine nukleare Eskalation faktisch vermieden worden ist, weil keine Seite ein Interesse an einer nuklearen Konfrontation gehabt habe. ${ }^{7}$ Ungeachtet dieser Kontroverse sind sich Optimisten und Pessimisten allerdings darin einig, dass das vordringliche Ziel in Südasien die Herstellung stabiler Abschreckung sein muss. Rüstungskontrolle kann dazu einen wichtigen Beitrag leisten. Nur fehlt in der Region bislang das technische Wissen und das politische Verständnis, dass Rüstungskontrolle Teil der Sicherheitspolitik ist und im nationalen Interesse beider Seiten liegt. ${ }^{8}$ Der Krieg um Kagil und die Krise von 2002 könnten aber als »Kuba-Erlebnis« Südasiens wirken und einen Prozess des »nuklearen Lernens« einleiten, der zur Einsicht in die Notwendigkeit kooperativer Rüstungssteuerung führt. Auch die USA und die Sowjetunion mussten 1962 erst bis an

4 Zum Problem der »opaken Proliferation« vgl. Benjamin Frankel, (Hrsg.), Opaque Nuclear Proliferation: Methodological and Political Implications, London 1991.

5 Peter R. Lavoy, South Asia, in: Jeffrey A. Larsen, Hrsg., Arms Control. Cooperative Security in a Changing Environment, Boulder 2002, 241-252, 247.

6 Glenn Snyder, Deterrence and Defense: Toward a Theory of National Security, Princeton 1961, 226; vgl. auch Frank C. Zagare/D. Marc Kilgour, Perfect Deterrence, Cambridge 2000, 189-192.

7 Vgl. Michael Krepon, The Stability-Instability Paradox, Misperception, and Escalation Control in South Asia, The Henry L. Stimson Center, May 2003.

8 Lavoy, op. cit., 246 
den Rand eines Nuklearkrieges gehen, um die Interdependenz ihrer Interessen angesichts atomarer Zerstörungsfähigkeit zu erkennen. ${ }^{9}$

Allerdings wird in Südasien dieser Lernprozess durch eine Reihe intervenierender Faktoren erschwert, weil die subregionale Bipolarität gleichsam »eingebettet « ist in regionale und globale Zusammenhänge. Diese bieten insbesondere Indien Anreize, an seinem militärischen Nuklearprogramm festzuhalten und lassen nur geringen Spielraum für Abrüstungsvorschläge, wie sie Pakistan wiederholt vorgetragen hat. Für Indien spielt nämlich neben Pakistan auch der »China-Faktor « eine wichtige Rolle. Beide Staaten waren in den vergangenen Jahrzehnten mehrfach militärisch miteinander in Konflikt geraten, und China wird als Groß- und Nuklearmacht teilweise sogar als größere Bedrohung für Indien wahrgenommen als Pakistan. Zweitens spielt für Indien auch der internationale Status eine wichtige Rolle, der mit dem Besitz von Nuklearwaffen verbunden ist. Indien war lange für die nukleare Abrüstung eingetreten und hat sich zur öffentlichen Demonstration seiner militärischen Nuklearkapazität erst entschlossen, als die Hoffnungen auf eine allgemeine nukleare Abrüstung mit der unbeschränkten Verlängerung des NPT 1995 verflogen waren. ${ }^{10}$ In der Bevölkerung Indiens wie Pakistans sind darüber hinaus Nuklearwaffen überaus populär. Sie gelten als Ausweis politischer Stärke und wirtschaftlich-technologischer Leistungsfähigkeit. Innenpolitisch werden deshalb nukleare Rüstungskontroll- und Abrüstungsschritte nur schwer zu vermitteln sein.

Diese Gemengelage aus rational-militärischen und psychologisch-politischen Motivationen für eine nukleare Bewaffnung macht die Einhegung dieser Rüstungsdynamik ausgesprochen kompliziert. Hinzu kommt, dass, wie in anderen Teilen Asiens auch, institutionelle Strukturen fehlen, die einen Dialog oder gar Verhandlungen zwischen den Parteien initiieren oder verstetigen könnten. Zwar haben Islamabad und New Delhi eine Reihe von »Vertrauensbildenden Maßnahmen« (CBMs = Confidence Building Measures) beschlossen, so etwa 1991 die Vereinbarung, den jeweiligen Luftraum nicht zu verletzen und Militärübungen und Truppenbewegungen entlang der gemeinsamen Grenze rechtzeitig anzukündigen. Doch sind diese Vereinbarungen widerholt verletzt worden, und auch der »Heiße Draht « zwischen den Obersten Heeresleitungen wurde missbraucht. ${ }^{11}$ Es gibt noch keine Tradition glaubwürdigen Verhandelns und noch kein Vertrauen in die gemeinsame Einsicht, dass nukleare Risikoreduktion im Interesse beider Seiten liegt. Auch die Regionalorganisation Südasiens, die South Asian Association of Regional Cooperation (SAARC) eignet sich kaum als Forum für einen sicherheitspolitischen Dialog; sie ist $\mathrm{zu}$ wenig entwickelt und leidet unter der ungleichen Größe und Stärke ihrer Mitglieder. Dennoch trafen sich Premierminister Vajpayee und Präsident Musharraf am

9 Vgl. Joseph S. Nye, Nuclear Learning and US-Soviet Security Regimes, in: International Organizations, Vol.41, No. 3, 371-402; George W. Breslauer/Philip E. Tetlock, (Hrsg.), Learning in US and Soviet Foreign Policy, Boulder 1991.

10 Vgl. dazu Christopher Daase, Der Anfang vom Ende des nuklearen Tabus. Zur Legitimitätskrise der Weltnuklearordnung, in: Zeitschrift für Internationale Beziehungen, 10. Jg., Heft 1, 7-41; Thazha V. Paul, The Systemic Bases of India's Challenge to the Global Nuclear Order, in: Nonproliferation Review, Vol. 6, No. 1, 1-11.

11 Lavoy, op. cit., 246.
Rande einer SAARC-Konferenz im Januar 2002, um die Entschärfung der Krise zu besprechen. Ob sich daraus ein Entspannungsdialog entwickelt, der zu rüstungskontrollpolitischen Ergebnissen führt, bleibt abzuwarten.

Südasien ist diejenige Sub-Region Asiens, in der die sicherheitspolitische Konstellation der des Kalten Krieges am ähnlichsten ist, weil sich mit Indien und Pakistan zwei Nuklearmächte gegenüberstehen. Deshalb können durchaus Erkenntnisse der Abschreckungstheorie und Erfahrungen nuklearer Rüstungskontrolle Anwendung finden. ${ }^{12}$ Nur ist regionale Abschreckung und Rüstungskontrolle weitaus schwieriger zu erzielen, weil außerregionale Faktoren die Konfliktdynamik ungünstig beeinflussen können. Gleichzeitig ist die Krisenstabilität aufgrund der geographischen Nähe der Kontrahenten geringer. Zur Stabilisierung der gegenwärtigen Lage und zur langfristigen Konfliktlösung müssen deshalb dringend Konzepte entwickelt werden, die allen drei Konfliktebenen der subregionalen zwischen Indien und Pakistan, der regionalen zwischen Indien und China, und der globalen zwischen den offiziellen und den nicht-offiziellen Nuklearmächten gerecht werden.

\section{Südostasien: Diffuse Multipolarität}

Im Gegensatz zu Südasien ist die Rüstungsdynamik in Südostasien auf konventionelle Waffensysteme beschränkt. Keiner der Staaten dieser Region besitzt Nuklearwaffen. 1994 haben sich alle zehn südostasiatischen Staaten sogar vertraglich verpflichtet, auch zukünftig auf Besitz, Erwerb und Stationierung von Nuklearwaffen zu verzichten, als sie Südostasien zu einer Nuklearwaffenfreien Zone (SEA NWFZ = South East Asia Nuclear Weapons Free Zone) erklärten. ${ }^{13}$

Aber auch in anderer Hinsicht unterscheidet sich Südostasien von anderen Regionen. Die strategische Situation ist nämlich weniger von einer regionalen Bipolarität, sondern mehr von einer diffusen Multipolarität geprägt. Drei Konfliktebenen haben dabei Einfluss auf Bedrohungswahrnehmungen und Rüstungsentscheidungen:

1. Innenpolitische Krisen und Konflikte, von denen Indonesien zurzeit am stärksten betroffen ist, das in vielen Landesteilen gleichzeitig mit Sezessionsbestrebungen konfrontiert ist. Aber auch Thailand, Myanmar, Malaysia und die Philippinen gehören zu den Staaten, die von ethnischen und religiösen Spannungen betroffen sind.

2. Auf zwischenstaatlicher Ebene sind eine Vielzahl von Territorialdisputen ungelöst, allen voran die Frage der Souveränitätsrechte über die Spratly-Inselgruppe im Südchinesischen Meer, die ganz oder in Teilen von Vietnam, den Philippinen, China und Taiwan sowie von Malaysia und Brunei beansprucht werden. Hinzu kommen nicht-militärische Sicherheitsprobleme wie Piraterie, Drogenhandel und

12 Vgl. Michael Krepon, Nuclear Risk Reduction: Is Cold War Experience Applicable for South Asia?, in: Michael Krepon/Chris Gagne, (Hrsg.), The Stability-Instability Paradox: Nuclear Weapons and Brinksmanship in South Asia, Report No. 38, The Henry Stimson Center, Washington, D.C. 2001, 1-14.

13 Vgl. Susanne Feske, Der Vertrag von Bangkok: Substanz oder Symbol? in: Christopher Daase/Constanze Eisenbart, (Hrsg.), Nuklearwaffenfreie Zonen. Neue Aktualität eines alten Konzepts, Heidelberg 2000, 137-159. 
Menschenschmuggel. Die Inseldispute und Piraterie haben Archipel- und Küstenstaaten wie Malaysia, Indonesien und Thailand in den letzten Jahren dazu veranlasst, ihre Marinen auszubauen und $\mathrm{zu}$ modernisieren. Aber auch weniger präzise zu benennende Faktoren wirken sich auf die Rüstungsdynamik aus: Konkurrenz um eine regionale Führungsrolle, ein generelles Misstrauen über die Intentionen der anderen Staaten und der Wettbewerb um Status und Prestige führen zu einem kompetitiven Rüstungsverhalten, das nicht dem gängigen Aktions-Reaktions-Schema oder dem Mechanismus des Sicherheitsdilemmas folgt.

3. Auf internationaler Ebene fühlen sich die Staaten der Region besonders nach der Reduzierung der amerikanischen Militärpräsenz von zunehmenden Großmachtrivalitäten bedroht. Zwar spielt nach dem Ende des Ost-WestKonflikts Russland keine bedeutende Rolle mehr, besonders seit es seine früheren Militärstützpunkte in Vietnam geräumt hat. Mit Sorge wird jedoch die Aufrüstung und Modernisierung der chinesischen Streitkräfte beobachtet. China gilt als potenziell expansive Großmacht, die traditionell Südostasien als ihre Einflusssphäre betrachtet und bereit ist, ihre Gebietsansprüche beispielsweise im Südchinesischen Meer auch militärisch durchzusetzen. Auch Japans zunehmend aktive militärische Rolle wird aufmerksam registriert. Mit Ausnahme Thailands litten alle südasiatischen Staaten unter der brutalen japanischen Besatzung vor und während des Zweiten Weltkrieges, und die Furcht vor einem remilitarisierten Japan wächst angesichts der schrittweisen Aufgabe der Zurückhaltung, die sich Japan militärisch und politisch selbst auferlegt hatte. Den südostasiatischen Staaten ist allerdings klar, dass sie selbst mit einer Bündelung all ihrer Militärpotenziale kein annäherndes Gleichgewicht weder mit China noch mit Japan herstellen oder ein glaubhaftes Abschreckungspotenzial aufbauen können. Dennoch versuchen sie, zumindest der chinesischen Marine eine effektive Seestreitmacht entgegenzusetzen.

Neben den genannten Faktoren gibt es noch eine andere entscheidende Variable, die Einfluss auf die Höhe der Militärausgaben und damit auf Rüstungsentscheidungen hat: die Höhe des verfügbaren Budgets. In Zeiten schlechter Konjunktur und besonders während der asiatischen Finanzkrise von 1997 gaben die Staaten weit weniger Geld für Militärgerät aus als in Wachstumsphasen. Umgekehrt wächst bei größerem Bruttoinlandsprodukt die Bereitschaft, in Rüstungsgüter zu investieren. Diesen Trend macht sich weltweit die Rüstungsindustrie zunutze, die nach der Sättigung heimischer Märkte offensiver auf den lange Zeit boomenden südostasiatischen Rüstungsmarkt drängt.

Im Gegensatz zu Südasien sind in Südostasien durchaus Institutionen vorhanden, die Mechanismen zur Rüstungskontrolle bereitstellen könnten. Die größte und älteste ist die 1967 gegründete ASEAN (Association of Southeast Asian Nations). Aus ganz unterschiedlichen Gründen vermied es die ASEAN jedoch, im multilateralen Rahmen Sicherheitsprobleme zu erörtern. Einerseits sollte gegenüber dem Antagonisten Vietnam nicht der Eindruck entstehen, dass es sich bei der ASEAN um ein Militärbündnis handelte, was Gegenmaßnahmen hätte provozieren können. Andererseits sollten Ähnlichkeiten mit der von den USA dominierten
SEATO (South East Asian Treaty Organization) vermieden werden. Deshalb gründete die ASEAN 1994 das ASEAN Regional Forum (ARF), dem neben den zehn ASEAN-Staaten auch Japan, Südkorea, Russland, China, die EU, Kanada, Australien und Neuseeland angehören. ${ }^{14}$ Die Notwendigkeit, die Rüstungsdynamik schon aus ökonomischen Gründen einzudämmen ebenso wie die Einsicht, dass bei mehr Transparenz von Rüstungsprogrammen nicht-bedrohungsbezogene Faktoren wie Konkurrenz oder Misstrauen erheblich reduziert werden könnten, fanden Eingang in die ehrgeizigen Ziele des ARF. Zum Spektrum vertrauensbildender Maßnahmen gehören der Austausch von Informationen über geplante Rüstungsbeschaffungen, die Veröffentlichung von Verteidigungsweißbüchern, die Ankündigung von Manövern, der Austausch von Manöverbeobachtern und präventive Diplomatie. $^{15}$

Eine Besonderheit des ARF ist die enge Verknüpfung von offizieller Diplomatie (Track 1) und inoffiziellen Dialogprozessen, die häufig von akademischen Institutionen oder Nichtregierungsorganisationen ausgehen (Track 2). Dadurch ist es gelungen, neue Ideen für vertrauensbildende Maßnahmen $\mathrm{zu}$ entwickeln und auch Probleme anzusprechen (z.B. den Spratly-Konflikt), die in offiziellen Verhandlungsforen abgeblockt worden wären. Insbesondere im Rahmen des CSCAP (Council of Security Cooperation in the Asia-Pacific), eines von amerikanischen Universitäten initiierten Dialogprozesses, sind informelle Verhandlungen geführt worden, die es etwa China erlaubt haben, sich schrittweise den multilateralen Verhandlungsmechanismen des ARF zu öffnen. ${ }^{16}$ Weit entfernt davon, nur eine »zahnlose Schwatzbude« zu sein, wie im Westen oft kritisiert wird, hat das ARF eine wichtige Sozialisierungfunktion, indem es - gerade aufgrund seiner geringen Institutionaliserung - Staaten die Möglichkeit bietet, informell und ohne Gesichtsverlust zu erkunden, »welches Kooperationsspiel gespielt wird «, um es politikwissenschaftlich auszudrücken. Aus Sicht der südostasiatischen Staaten ist das ARF deshalb selbst bereits eine vertrauensbildende Maßnahme, auch wenn die Implementation der substantiellen Vereinbarungen noch zu wünschen übrig lässt.

Die schleppende Umsetzung von militärischen Kooperationsvereinbarungen hat wiederum historische und kulturelle Ursachen, denn es gibt in der Region keine Tradition im Bereich gegenseitiger Information über Rüstungsprogramme und Militärstrategien. Sicherheit - innere und äußere - gilt nach wie vor als sensibler Bereich, in den man externen Parteien nur ungern Einblick gewährt, weil dieser Einblick alleine schon $\mathrm{zu}$ einem Sicherheitsverlust führen könnte. Rüstungskontrolle im Sinne von Abrüstung ist zudem angesichts knapper Ressourcen nahezu undenkbar - kein Staat wird einmal erworbene Rüstungsgüter verschrotten. Folglich sollten die Erwartungen an Rüstungskontrolle nicht zu hoch gesteckt werden. In Südostasien kommt es deshalb zunächst

14 Vgl. Michael Leifer, The ASEAN Regional Forum: Extending ASEAN's Model of Regional Security, Adelphi Papers No. 302, Oxford 1996.

15 Vgl. Ralph Cossa, (Hrsg.), Confidence and Security Building in the Asia-Pacific, Washington, D.C. 1995.

16 Alastair Iain Johnston, The Myth of the ASEAN Way? Explaining the Evolution of the ASEAN Regional Forum, in: Helga Haftendorn/Robert O. Keohane/Celeste A. Wallander, (Hrsg.), Imperfect Unions. Security Institutions over Time and Space, Oxford 1999, 287-324, 303. 
darauf an, einen kontrollierten und miteinander abgestimmten Prozess der Rüstungsentscheidungen einzuleiten, bei dem gemeinsame Interessen identifiziert, Ressourcen wirtschaftlicher eingesetzt, gegenseitige Bedrohungswahrnehmungen im Vorfeld vermieden bzw. abgebaut und Fehlentscheidungen verhindert werden. Die Verstetigung des sicherheitspolitischen Dialogs könnte somit zu Verhaltens- und Erwartungssicherheit beitragen und Vertrauen bilden und festigen.

\section{Nordostasien: Asymmetrische Rivalität}

Nordostasien bietet rüstungskontrollpolitisch wieder ein ganz anderes Bild. In dieser Subregion treten nahezu alle Probleme der Aufrüstung und des Rüstungswettlaufs auf: China als Nuklearmacht, Nordkoreas nukleares Potenzial, Japans nukleare Option, eine konventionelle Aufrüstung Nord- und Südkoreas, die Expansion des chinesischen Militärpotenzials, die von den USA geplante Einrichtung einer regionalen Raketenabwehr (TMD) in Taiwan, Südkorea und Japan, die Proliferation konventioneller und nuklearer Technologien durch Nordkorea in Krisengebiete sowie die konventionelle Aufrüstung Japans, das mittlerweile Rang 3 auf der Weltrangliste der Länder mit den höchsten Militärausgaben einnimmt (obwohl es offiziell nur $1 \%$ seines BSP für Rüstung ausgibt).

Im Zentrum der internationalen Aufmerksamkeit steht seit Jahren das nordkoreanische Atomwaffenprogramm. ${ }^{17}$ Obwohl Pjöngjang 1985 dem NPT beitrat, verhinderte es bis 1992 erfolgreich Inspektionen durch die internationale Atomenergiebehörde (IAEA = International Atomic Engergy Agency). Als diese schließlich herausfand, dass Nordkorea Plutonium - vermutlich für den Bau von Nuklearwaffen - abgezweigt hatte, erklärte Pjöngjang 1993 seinen Austritt aus dem NPT. Die Krise konnte erst beigelegt werden als die USA im Oktober 1994 im sogenannten »Agreed Framework« für Nordkoreas Verbleib im NPT und die Suspendierung des Nuklearprogrammes die Lieferung zweier proliferationsresistenter Leichtwasserreaktoren zusagte. Die Vereinbarungen wurden von Nordkorea allerdings nicht eingehalten, und die Spannungen mit den USA verschärften sich angesichts des nordkoreanischen Raketenprogramms. ${ }^{18}$ Am 10. Januar 2003 zog sich Pjöngjang endgültig aus dem NPT zurück, nachdem es im Oktober 2002 die Existenz eines zweiten geheimen Nuklearwaffenprogramms auf der Basis hochangereicherten Urans eingeräumt hatte. ${ }^{19} \mathrm{Zu}$ dieser Eskalation hatte nicht unwesentlich die Aufgabe der »Zuckerbrot- und-PeitschePolitik « der USA und die eindeutig auf Regime-Wechsel zielende Rhetorik der Bush-Administration geführt.

Die Dramatik dieses Konflikts hat eine Reihe anderer Sicherheitsprobleme in den Hintergrund rücken lassen, die Nordostasien gefährden. Es handelt sich dabei größtenteils um Territorialkonflikte, wie z.B. den Streit um die Kurilen-Inseln

17 Vgl. Larry A. Niksch, North Korea's Nuclear Weapons Program, Congressional Research Service, Issue Brief, Washington, D.C. 2002; Victor D. Cha, North Korea's Weapons of Mass Destruction. Badges, Shields, or Swords?, in: Political Science Quarterly, Vol. 117, No. 2, 209-230.

18 Vgl. Sebastian Harnisch, Das nordkoreanische Raketenprogramm. Gespräche zu dessen Beendigung zwischen Washington und Pjöngjang, in: Österreichische militärische Zeitschrift, Jg. 40, Heft 4, 415-424.

19 Kay Möller, Pjöngjang bekennt sich zur Bombe. Ende der internationalen Einbindungspolitik, SWP-aktuell 41, Berlin 2002. zwischen Japan und Russland. Dieser Konflikt verhindert noch immer einen Friedensvertrag zwischen beiden Staaten, die sich technisch gesehen auch sechzig Jahre nach Kriegsende noch immer im Kriegszustand befinden. Auch an anderen Stellen sind durch die Genfer Seerechtskonvention von 1982 exklusive Wirtschaftszonen $(\mathrm{EEZ}=$ Exclusive Economic Zones) entstanden, die sich überlappen und damit zu Streitigkeiten über die Zugehörigkeit von Inseln und Inselgruppen führen. Dazu gehören die von China und Japan beanspruchten Diayu (jap.: Senkaku)-Inseln sowie die von Südkorea und Japan beanspruchten Tokto- bzw. Takeshima-Inseln. Ferner führen die Soveränitätskonflikte zwischen Nord- und Südkorea sowie zwischen der Voksrepublik China und Taiwan in regelmäßigen Abständen zu militärischen Zwischenfällen und Provokationen und erhöhen so das Instabilitätspotenzial in der gesamten asiatisch-pazifischen Region. Die Bereitschaft zur gewaltsamen Konfliktaustragung ist bei einigen Staaten, namentlich Nordkorea und der Volksrepublik China, erklärtermaßen hoch.

Im Hintergrund lauern allerdings auch Großmachtkonflikte. Neben den immer wieder aufkeimenden Großmachtgelüsten Japans ist es vor allem Chinas Anspruch auf eine regionale Hegemonialposition, die die Staaten in der Region verunsichert. Langfristig dürfte jedoch entscheidend sein, wie sich das Verhältnis zwischen China und den USA entwickelt. Die amerikanischen Pläne für eine regionale Raketenabwehr (Theater Missile Defense $=$ TMD) in Kooperation mit Taiwan, Südkorea und Japan haben das Verhältnis deutlich verschlechtert. Rüstungskontrollgespräche könnten hier entspannende Wirkung haben und sich positiv auf die regionalen und die globalen Beziehungen auswirken. ${ }^{20}$

Doch für derartige Initiativen fehlt auch in Nordostasien einstweilen die institutionelle Basis. Die auf staatlicher Ebene vorhandenen Institutionen wie APEC (Asian Pacific Economic Cooperation) und KEDO (Korean Peninsular Energy Development Organisation) verstehen sich in erster Linie als Institutionen zur Förderung der Wirtschaftsbeziehungen. Nichtsdestotrotz haben sie am Rande auch sicherheitspolitische Relevanz. Dies belegt eine weitere Besonderheit der spezifischen Form von Rüstungssteuerung in Asien: die Vermischung von wirtschaftlichen, politischen und militärischen Sachthemen. So sollten beispielsweise im Rahmen von KEDO wirtschaftliche und energiepolitische Anreize geschaffen werden, um Nordkorea von der Verfolgung seiner Nuklearpläne abzubringen. Solche »Paketlösungen« werden vielfach auf bilateraler Ebene ausgehandelt und dann multilateral »verpackt «. Mitglieder von KEDO sind dementsprechend 13 Staaten, darunter, neben den USA, Japan und Südkorea, auch Staaten wie Polen, Neuseeland, Chile und die Tschechische Republik. Bei allem guten Willen kann das aber nicht darüber hinwegtäuschen, dass in Nordostasien multilaterale Kooperationsstrukturen wie in Europa fehlen, auf deren Grundlage Rüstungskontrollgespräche sich entwickeln könnten.

Als Ersatz dienen auch hier, wie in Südostasien, Track-TwoInstitutionen. Neben dem bereits erwähnten CSCAP ist hier

20 Brad Roberts, East Asia, in: Jeffrey A. Larsen, (Hrsg.), Arms Control. Cooperative Security in a Changing Environment, Boulder 2002, 253 267, 263. 
vor allem der »Northeast Asia Cooperation Dialogue « (NEACD) zu nennen..$^{21}$ Der NEACD wurde 1993 gegründet und konzipierte auf seinen jährlichen Treffen die Idee der »Mutual Reassurance Measures «. Dahinter verbirgt sich mehr als die Weigerung, das westliche Konzept von vertrauensbildenden Maßnahmen zu übernehmen. Vielmehr geht es auch hier nicht nur um rein militärische Kooperationsmaßnahmen, sondern auch um die Einbeziehung von wirtschaftlichen und energiepolitischen Fragen. Als wichtigen Erfolg konnte NEACD 2002 die Teilnahme einer Delegation aus Nordkorea vermelden, das zwar zu den Gründungsmitgliedern von NEACD gehörte, aber den jährlichen Treffen bislang ferngeblieben war. Entscheidend bei dieser Form von Kooperation und Verhandlung ist freilich, dass ab einer bestimmten Phase des informellen Prozesses die Vermittlung an die staatliche Ebene gelingt. Während bei der CSCAP dies durch die enge Anbindung an das ARF gewährleistet zu sein scheint, gestaltet sich die Umsetzung in offizielle Politik für NEACD schwieriger.

\section{Fazit}

In keiner anderen Weltregion hat das Ende des Kalten Krieges die Notwendigkeit für Rüstungskontrolle stärker akzentuiert als in Asien. ${ }^{22}$ Doch traditionelle Konzepte und Theorien kooperativer Rüstungssteuerung sind hier nur von begrenztem Nutzen. Während Versatzstücke der Theorie der

21 http://www-neacd.ucsd.edu/

22 James J. Wirtz, Conclusion: The Future of Arms Control, in: Jeffrey A. Larsen, (Hrsg.), Arms Control. Cooperative Security in a Changing Environment, Boulder 2002, 343-351, 347.
Abschreckung, Vertrauensbildung, Krisenstabilität usw. ihren heuristischen Wert behalten, sind die regionalen und subregionalen Bedingungen so spezifisch, dass sie nicht eins-zu-eins übertragen werden können. Vielmehr ist eine doppelte Anpassung notwendig: einerseits an die konfliktspezifischen subregionalen Strukturen, andererseits an die kulturspezifischen regionalen Bedingungen.

Wir haben in Asien drei dominierende Konfliktstrukturen identifiziert: die eingebettete Bipolarität in Südasien, die diffuse Multipolarität in Südostasien und die asymmetrische Rivalität in Nordostasien. Diese Konstellationen unterscheiden sich von der globalen Bipolarität des Kalten Krieges und erfordern daher neue Konzepte der Rüstungskontrolle. Darüber hinaus haben wir regionalspezifische Besonderheiten festgestellt, die Rüstungskontrolle in Asien erschweren: das Fehlen bzw. die nur schwache Ausprägung sicherheitspolitischer Institutionen, das Misstrauen gegenüber multilateralen Verhandlungsforen und eine Rüstungsdynamik, in der sich nicht immer klare Konfliktlinien und Aktions-ReaktionsMuster erkennen lassen. Dies hat zur Folge, dass es keine Block-zu-Block-Verhandlungen geben kann. Zudem ist die Rüstungsdynamik vielfach eng verbunden mit wirtschaftlichen Fragen. Rüstungssteuerung funiktioniert daher nur unter Einbeziehung ökonomischer Aspekte. Und schließlich herrscht eine andere Kooperationskultur, in der informelle Absprachen, Dialog und Vertrauensbildung mehr zählen als hartes Verhandeln und formalisierte Verträge. Der Erfolg dieser Form von Rüstungssteuerung, bei der die enge Verbindung von Track-One und Track-Two-Diplomatie eine wichtige Rolle spielt, kann nur in zweiter Linie an ihrem Output gemessen werden. In erster Linie zählt, dass überhaupt Gespräche und Verhandlungen stattfinden.

\section{Politische Handlungsmöglichkeiten zur Kontrolle biologischer Waffen*}

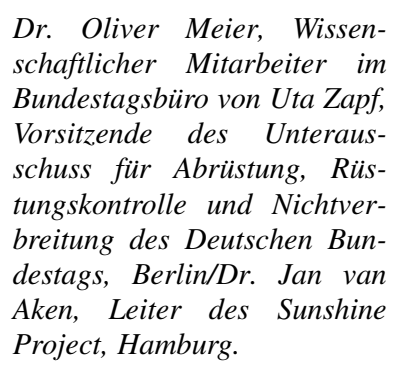

\section{Einleitung}

Biologische Waffen (BW) sind eine der größten Bedrohungen für die internationale Sicherheit. Während diese Bedrohung längst im 21. Jahrhundert angekommen ist, befindet sich die internationale Kontrolle der B-Waffen immer noch auf dem Stand von 1972. Seinerzeit wurde das multilaterale Biowaffenübereinkommen (BWÜ) vereinbart. Als sich im August 2003 - rund 30 Jahre nach Zeichnung der Biowaffenkonvention - Experten im Rahmen des
BWÜ in Genf trafen, wurde die Lücke zwischen Bedrohung und politischem Handeln erneut deutlich. Ziel des Treffens war nicht die Aushandlung dringend benötigter völkerrechtlich verbindlicher, strenger Kontrollmaßnahmen, wie sie für nukleare und chemische Waffen seit langem üblich sind. Stattdessen beschränkten sich die Diplomaten auf den Austausch von Details über nationale Gesetze und Regelungen zur sicheren Handhabung von Mikroorganismen. Neue internationale Vereinbarungen standen nicht einmal auf der Tagesordnung. ${ }^{1}$

An dieser unbefriedigenden Situation dürfte sich in den nächsten Jahren kaum etwas ändern. Multilaterale Verhandlungen über einen Verifikationsmechanismus für das BWÜ 